\title{
Melón (Cucumis melo L.) Honey Dew cultivado bajo invernadero: correlación entre densidad de siembra y variables de rendimiento
}

\section{Honey Dew melon (Cucumis melo L.) grown under greenhouse conditions: correlation among plant density and yield variables}

Jorge Manuel Díaz-Alvarado', José Eladio Monge-Pérez², Michelle Loría-Coto ${ }^{3}$

Fecha de recepción: 12 de marzo de 2020

Fecha de aprobación: 23 de julio de 2020

Díaz-Alvarado, J.M; Monge-Pérez, J.E; Loría-Coto, M. Melón (cucumis melo I.) Honey dew cultivado bajo invernadero: correlación entre densidad de siembra y variables de rendimiento. Tecnología en Marcha. Vol. 34-3 Julio-Setiembre 2021. Pág 34-50.

doi) https://doi.org/10.18845/tm.v34i3.5033

1 Ingeniero agrónomo. Amazon Development Center Germany GmbH. Alemania. Correo electrónico: jorgeda@gmail.com

2 Ingeniero agrónomo. Estación Experimental Agrícola Fabio Baudrit Moreno y Sede de Guanacaste, Universidad de Costa Rica, Costa Rica. Correo electrónico: melonescr@yahoo.com.mx

3 Economista agrícola. Escuela de Ciencias Exactas y Naturales, Universidad Estatal a Distancia, Costa Rica. Correo electrónico: michelle loria@yahoo.com 


\title{
Palabras clave
}

Cucumis melo; número de frutos por planta; rendimiento; correlación de Pearson; regresión lineal; sólidos solubles totales

\section{Resumen}

Se establecieron correlaciones de Pearson en melón Honey Dew producido bajo invernadero, manejado con tres tipos de poda ( 1 tallo; 2 tallos; sin poda), entre la densidad de siembra y las siguientes variables: edad al inicio de la cosecha, peso del fruto, número de frutos por planta y por metro cuadrado, rendimiento por planta y por área, firmeza de la pulpa del fruto, y porcentaje de sólidos solubles totales. El cultivo se manejó con fertirrigación, en sustrato de fibra de coco. Para los tres tipos de poda, las correlaciones de Pearson que tuvieron significancia estadística $(p \leq 0,05)$, en cuyos casos se obtuvo la regresión lineal, fueron entre la densidad de siembra y: número total de frutos por planta $\left(r=-0,65^{\star \star}\right)$; número de frutos comerciales por planta $\left(r=-0,65^{\star \star}\right)$; rendimiento total por planta $\left(r=-0,69^{\star *}\right)$; y rendimiento comercial por planta $\left(r=-0,69^{\star *}\right)$. Para los tratamientos con poda a 2 tallos, se obtuvo una correlación significativa entre la densidad de siembra y el porcentaje de sólidos solubles totales $\left(r=-0,64^{\star}\right)$. Para el tratamiento sin poda, se obtuvo una correlación significativa entre la densidad de siembra y la edad al inicio de la cosecha $\left(r=0,98^{* *}\right)$. Se concluye que, en melón Honey Dew manejado con cualquiera de los tipos de poda evaluados, el uso de una mayor densidad de siembra provoca una disminución en el número de frutos (totales y comerciales) por planta, y en el rendimiento total y comercial por planta.

\section{Keywords}

Cucumis melo; number of fruits per plant; yield; Pearson correlation; linear regression; total soluble solids.

\begin{abstract}
For Honey Dew melon grown under greenhouse conditions and managed with three pruning systems (1-stem; 2-stem; no pruning), the researchers estimated Pearson correlations among plant density and the following variables: days to start of harvest, fruit weight, number of fruits per plant and per square meter, yield per plant and per area, fruit flesh firmness and percentage of total soluble solids. The crop was planted on coconut fiber as substrate, and managed with fertigation. For all three pruning systems, the Pearson correlations that were statistically significant $(p \leq 0,05)$, were obtained between plant density and: total number of fruits per plant $(r$ $\left.=-0,65^{\star \star}\right)$; number of commercial fruits per plant $\left(r=-0,65^{\star \star}\right)$; total yield per plant $\left(r=-0,69^{\star *}\right)$; and commercial yield per plant $\left(r=-0,69^{\star \star}\right)$; in those cases the linear regression was calculated. For 2-stem pruning treatments, a significant correlation was obtained between plant density and percentage of total soluble solids $\left(r=-0,64^{\star}\right)$. For no pruning treatment, a significant correlation was obtained between plant density and days to start of harvest $\left(r=0,98^{\star \star}\right)$. It is concluded that in Honey Dew melon managed with any of the evaluated pruning systems, the use of a higher plant density lead to a decrease in the number of fruits (total and commercial) per plant, and in the total and commercial yield per plant.
\end{abstract}




\section{Introducción}

El melón (Cucumis melo L.) es uno de los cultivos económicamente más importantes en Costa Rica. Esta especie pertenece a la familia Cucurbitaceae, y presenta una planta herbácea, anual, cuyos tallos pueden ser rastreros o trepadores si se les facilita un tutorado [1] [2]. Los principales tipos de melón que se cultivan en este país son: Harper, Amarillo, Cantaloupe, Honey Dew, Galia, Charentais, Piel de Sapo, y Orange Flesh [3].

El cultivo protegido presenta varias ventajas sobre el cultivo a campo abierto, como la obtención de mayor número de cosechas durante el año, precocidad de la cosecha, economía de agua y fertilizantes, mayor rendimiento, y mejor calidad de los frutos [4]. En el cultivo bajo ambiente protegido se alteran las características ambientales de clima y de suelo: hay menor radiación solar global, evapotranspiración y viento, y hay mayor radiación difusa, temperatura y humedad relativa del aire [5].

Por otra parte, el cultivo de melón en sistema hidropónico permite un control parcial de las condiciones climáticas, menor aplicación de plaguicidas, manejo adecuado del agua y de los nutrientes de acuerdo al desarrollo del cultivo, la posibilidad de cultivar a mayor densidad, y un aumento significativo en la productividad y calidad del producto final [4].

Una ventaja de la producción de melón en invernadero es que se puede cultivar durante todo el año. En Costa Rica hay una importante demanda insatisfecha durante la época lluviosa, debido a la muy baja o nula producción (y de baja calidad) de melón a campo abierto, por lo que los consumidores podrían estar dispuestos a pagar precios altos por frutos de alta calidad en esa época; la producción de melón bajo ambiente protegido abriría esta oportunidad comercial para los agricultores [6] [7].

La calidad de los frutos de melón está relacionada con características como la concentración de sólidos solubles totales, la apariencia interna y externa del fruto, el grosor de la pulpa y el sabor, las que determinan la aceptabilidad del consumidor [4].

Otra ventaja es que se podrían obtener mejores rendimientos en comparación con los obtenidos a campo abierto, pues permite prolongar el ciclo productivo del cultivo [2] [7] [8]. Para garantizar un buen rendimiento y calidad en invernadero se pueden utilizar tres prácticas importantes: un sistema de amarre o tutorado de las plantas (con cuerdas o mallas), una mayor densidad de siembra, y un sistema de podas [9].

Varios investigadores han estudiado el efecto de la densidad de siembra sobre el cultivo de melón en invernadero; generalmente el rendimiento aumenta al utilizar una mayor densidad [10] [11] [12] [13] [14], y a veces esto provoca un menor peso del fruto [10] [13], pero en otras ocasiones no se afecta el peso o la calidad del mismo [12] [13] [14].

En cuanto a la implementación de sistemas de podas, lo que se busca es adelantar la cosecha, basándose en el principio botánico de que en melón las flores femeninas o hermafroditas sólo aparecen en las ramas secundarias o terciarias [1] [15] [2]. Existen muchos sistemas de podas en melón, pero generalmente lo que se busca es mantener un balance para disminuir el vigor vegetativo y adelantar la aparición de flores femeninas o hermafroditas. Las prácticas de poda involucran la eliminación o conservación del tallo principal, y el manejo de uno, dos o más tallos secundarios y terciarios [2].

La existencia de correlaciones significativas entre diversas variables en un cultivo puede ayudar a realizar una selección más rápida de los mejores genotipos a nivel de producción agrícola, y esto es también un factor de relevancia en los procesos de fitomejoramiento, pues ayuda a identificar las variables más importantes a ser consideradas para la generación de nuevos genotipos [16]. 
El objetivo de esta investigación fue establecer correlaciones de Pearson entre la densidad de siembra y variables de rendimiento, en melón Honey Dew cultivado en invernadero, en Alajuela, Costa Rica.

\section{Materiales y métodos}

La siembra se realizó en condiciones hidropónicas, en el invernadero de Hortalizas de la Estación Experimental Agrícola Fabio Baudrit Moreno (EEAFBM), la cual está localizada en Barrio San José de Alajuela, Costa Rica, a una altitud de 883 msnm.

Se sembró el híbrido de melón tipo Honey Dew JMX-701 F-1 (Cucumis melo L. var. inodorus). Las plántulas se trasplantaron el 17 de abril del 2012, cuando tenían dos hojas verdaderas expandidas.

El cultivo se realizó en sacos de fibra de coco, de $1 \mathrm{~m}$ de largo, $20 \mathrm{~cm}$ de ancho y $15 \mathrm{~cm}$ de altura. Las plantas se sujetaron por medio de dos mallas plásticas, ubicadas una a cada lado de cada hilera de plantas.

Se evaluaron nueve tratamientos, correspondiente a la combinación factorial de tres densidades de siembra y tres tipos de poda (cuadro 1). La distancia entre hileras fue de 1,54 m, y la distancia entre plantas fue de 33,0; 20,0; y 16,7 cm, correspondientes a una densidad de siembra de 1,$95 ; 3,25$; y 3,90 plantas $/ \mathrm{m}^{2}$, respectivamente. Los tipos de poda fueron: un tallo secundario; dos tallos secundarios; y plantas sin poda. La poda del tallo principal se realizó a los 15 días después del trasplante (ddt), cuando las plantas tenían tres hojas verdaderas expandidas [9], y se seleccionó uno o dos tallos secundarios, según el tratamiento correspondiente. El tratamiento sin poda se dejó a libre crecimiento.

Para todos los tratamientos, los tallos principales o secundarios fueron tutorados en una malla para el soporte de hortalizas, de polietileno, de 2,0 m de altura. En los tratamientos con poda, una vez tutorado el tallo secundario se realizaron podas semanales de la siguiente manera: los tallos terciarios que tenían fruto se podaron después de la segunda hoja emergida posterior al fruto, y los tallos terciarios sin fruto se podaron después de la cuarta hoja emergida [9]; esta poda de mantenimiento fue realizada durante los 22 días posteriores a la poda inicial, y a partir de ese momento no fue necesario realizar más podas, debido al escaso desarrollo de nuevos tallos terciarios.

Cuadro 1. Tratamientos evaluados.

\begin{tabular}{|c|c|c|}
\hline Tratamiento & Densidad $\left(\right.$ plantas $\left./ \mathrm{m}^{2}\right)$ & Tipo de poda \\
\hline 1 & 1,95 & 1 tallo \\
\hline 2 & 1,95 & 2 tallos \\
\hline 3 & 1,95 & Sin poda \\
\hline 4 & 3,25 & 1 tallo \\
\hline 5 & 3,25 & 2 tallos \\
\hline 6 & 3,25 & Sin poda \\
\hline 7 & 3,90 & 1 tallo \\
\hline 8 & 3,90 & 2 tallos \\
\hline 9 & 3,90 & Sin poda \\
\hline
\end{tabular}


Se implementó un sistema de manejo integrado de plagas, y se utilizó un programa de fertilización validado para la producción comercial de melón, según las experiencias previas en el invernadero de la EEAFBM. El fertirriego se suministró a cada hora, entre las 7:00 a.m. y las 4:00 p.m. Al inicio de la floración se introdujo una colmena de abejas (Apis mellifera) con el fin de promover una adecuada polinización.

El periodo total de cultivo fue hasta el 3 de agosto de 2012 (108 ddt); en los tratamientos más precoces la cosecha inició el 2 de julio de 2012 (76 ddt), y se extendió por un periodo de 32 días. El índice de cosecha se basó en la apariencia externa del fruto, donde se consideraron aptos para cosecha los frutos que presentaban una coloración típica de madurez (color crema claro, sin brillo), y un anillo de color amarillo en la zona de la unión peduncular. Se consideró como fruto de calidad comercial aquél con un peso superior a $599 \mathrm{~g}$.

Se evaluaron las siguientes variables:

1. Edad al inicio de la cosecha (ddt): se registró el tiempo transcurrido entre el trasplante y el inicio de la cosecha, para cada tratamiento.

2. Número de frutos por planta y por metro cuadrado: se obtuvo al registrar el número total de frutos producidos en cada parcela, y se dividió ese dato entre el número de plantas por parcela; y luego se multiplicó el valor obtenido por la densidad de siembra.

3. Rendimiento por planta (g) y por área (ton/ha): se estimó el peso de los frutos de melón producidos en cada parcela, y se dividió ese dato entre el número de plantas por parcela; y luego se multiplicó el valor obtenido por la densidad de siembra.

4. Peso del fruto (g): se midió el peso de cada fruto producido, y se obtuvo el promedio.

5. Porcentaje de sólidos solubles totales ( ${ }^{\circ}$ Brix): se obtuvo el porcentaje de sólidos solubles totales de cada fruto, y se obtuvo el promedio.

6. Firmeza de la pulpa del fruto (N): se midió la firmeza de la pulpa de cada fruto, y se obtuvo el promedio.

El peso de los frutos se obtuvo con una balanza electrónica marca Ocony, modelo TH-I-EK, de 5000,0 \pm 0,1 $\mathrm{g}$ de capacidad. El porcentaje de sólidos solubles totales se determinó con un refractómetro manual marca Atago, modelo $\mathrm{N}-1 \mathrm{a}$, con una capacidad de 32,0 \pm 0,2 ${ }^{\circ}$ Brix. Para la evaluación de firmeza del fruto se utilizó un penetrómetro portátil marca Effegi, modelo FT-327, con una capacidad de 12,5 \pm 0,1 kilogramo-fuerza, y los datos se multiplicaron por un factor de conversión de 9,806 para obtener el dato en Newtons (N); se utilizó el puntero cuya base mide $7,5 \mathrm{~mm}$ de ancho.

Se utilizó un diseño experimental irrestricto al azar, con arreglo de parcelas divididas; las parcelas grandes correspondieron a las densidades de siembra, y las pequeñas a los diferentes sistemas de podas. Se establecieron cuatro repeticiones por tratamiento. El área útil fue equivalente a dos metros lineales $\left(3,1 \mathrm{~m}^{2}\right)$ a lo largo de la hilera de siembra. La cantidad de plantas incluidas en cada parcela útil varió en función de la densidad de siembra: la densidad de 1,95 plantas $/ \mathrm{m}^{2}$ tuvo 6 plantas; la densidad de 3,25 plantas $/ \mathrm{m}^{2}$ tuvo 10 plantas; y la densidad de 3,90 plantas $/ \mathrm{m}^{2}$ tuvo 12 plantas. Todos los frutos producidos dentro de la parcela útil fueron evaluados.

Se obtuvo el coeficiente de correlación de Pearson $(r)$ entre la densidad de siembra y las variables de rendimiento, y para aquellas combinaciones en que se obtuvo significancia estadística ( $p \leq 0,05)$, se calculó la regresión lineal con su respectiva ecuación y su coeficiente de determinación $\left(R^{2}\right)$. Las correlaciones se obtuvieron tanto entre el total de tratamientos evaluados, como según el tipo de poda. 


\section{Resultados y discusión}

Durante el desarrollo de este ensayo, la temperatura promedio dentro del invernadero fue de 25 ${ }^{\circ} \mathrm{C}$, con un rango entre 17 y $41^{\circ} \mathrm{C}$; y la humedad relativa promedio fue de $78 \%$, con un rango entre 24 y $100 \%$.

Con respecto a la correlación entre la densidad de siembra y el número total de frutos por planta, el valor obtenido fue negativo y altamente significativo para el total de tratamientos $(r=$ $\left.-0,65 ; R^{2}=0,42\right)$ (cuadro 2, figura 1), así como para el tratamiento sin poda $\left(r=-0,75 ; R^{2}=0,56\right.$ ) (figura 2); y fue significativo para la poda a 1 tallo $\left(r=-0,66 ; R^{2}=0,43\right)$ (figura 3$)$ y a 2 tallos $(r$ $=-0,62 ; R^{2}=0,39$ ) (figura 4).

Las plantas del híbrido JMX-701 F-1 son bastante vigorosas en su desarrollo vegetativo, y de rápido crecimiento, en comparación con otros genotipos, lo que provoca una importante competencia por recursos (luz, espacio, agua, nutrientes) entre los individuos de una población, y es posiblemente por esta razón que disminuyó la cantidad de frutos por planta conforme aumentó la densidad.

Varios autores también obtuvieron un menor número total de frutos por planta al aumentar la densidad de siembra en melón [12] [11] [17], al igual que los resultados hallados en el presente ensayo. Sin embargo, otros investigadores no encontraron diferencias en el número total de frutos por planta al evaluar diferentes densidades de siembra [18] [13] [19] [14].

Cuadro 2. Coeficientes de correlación de Pearson entre densidad de siembra y número total de frutos por planta en melón Honey Dew.

\begin{tabular}{|c|c|c|c|}
\hline Tratamiento & $\begin{array}{c}\text { Coeficiente de } \\
\text { correlación }(r)\end{array}$ & Probabilidad $(p)$ & $\begin{array}{c}\text { Coeficiente de determinación de la } \\
\text { regresión lineal }\left(R^{2}\right)\end{array}$ \\
\hline Total & $-0,65$ & $* \star$ & 0,42 \\
\hline Poda a 1 tallo & $-0,66$ & $*$ & 0,43 \\
\hline Poda a 2 tallos & $-0,62$ & $*$ & 0,39 \\
\hline Sin poda & $-0,75$ & $* \star$ & 0,56 \\
\hline
\end{tabular}

Nota: $n s=$ no significativo; ${ }^{*}=$ significativa $(p \leq 0,05) ;{ }^{* *}=$ altamente significativa $(p \leq 0,01)$.

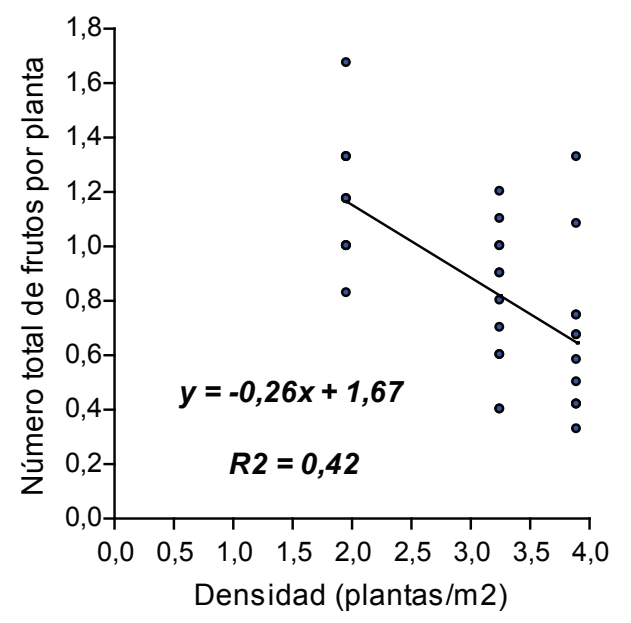

Figura 1. Regresión lineal del número total de frutos por planta versus la densidad de siembra para el total de tratamientos evaluados. 


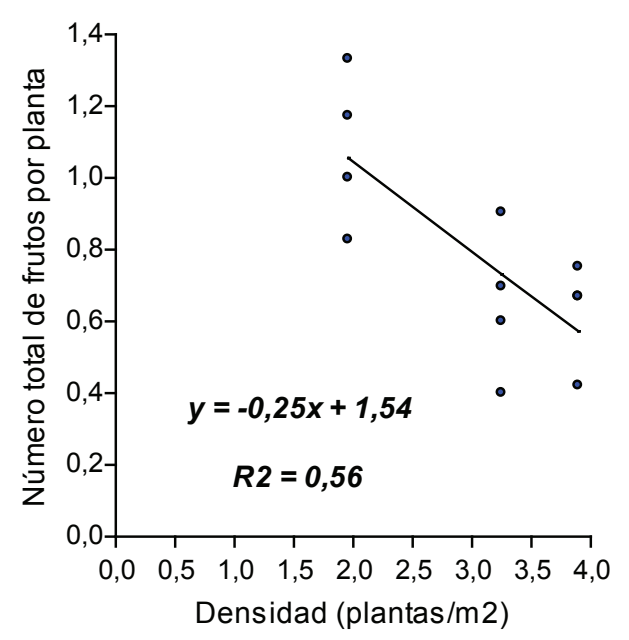

Figura 2. Regresión lineal del número total de frutos por planta versus la densidad de siembra para los tratamientos sin poda.

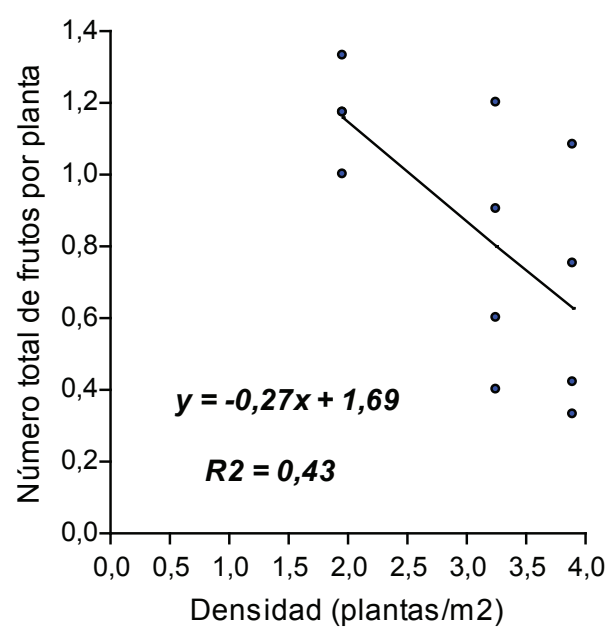

Figura 3. Regresión lineal del número total de frutos por planta versus la densidad de siembra para los tratamientos con poda a 1 tallo.

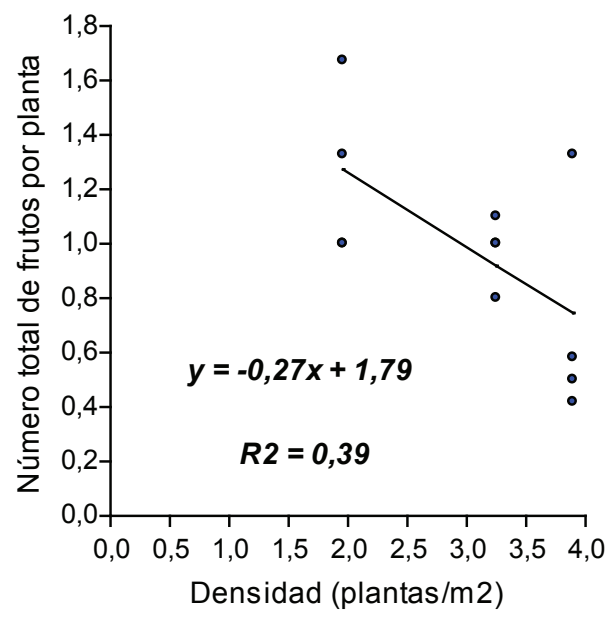

Figura 4. Regresión lineal del número total de frutos por planta versus la densidad de siembra para los tratamientos con poda a 2 tallos. 
La correlación entre la densidad de siembra y el número de frutos comerciales por planta fue negativa y altamente significativa, tanto para el total de tratamientos $\left(r=-0,65 ; R^{2}=0,42\right)$ (cuadro 3, figura 5), como para el tratamiento sin poda $\left(r=-0,78\right.$; $R^{2}=0,62$ ) (figura 6); y fue significativa para los tratamientos con poda a 1 tallo $\left(r=-0,62 ; R^{2}=0,38\right)$ (figura 7 ) y con poda a 2 tallos ( $\left.r=-0,66 ; R^{2}=0,44\right)$ (figura 8$)$.

Sin embargo, otros autores no hallaron diferencias en el número de frutos comerciales por planta, al evaluar diferentes densidades de siembra en melón tipo Cantaloupe [19].

Cuadro 3. Coeficientes de correlación de Pearson entre densidad de siembra y número de frutos comerciales por planta en melón Honey Dew.

\begin{tabular}{|c|c|c|c|}
\hline Tratamiento & $\begin{array}{c}\text { Coeficiente de } \\
\text { correlación }(r)\end{array}$ & Probabilidad $(p)$ & $\begin{array}{c}\text { Coeficiente de determinación de la } \\
\text { regresión lineal }\left(R^{2}\right)\end{array}$ \\
\hline Total & $-0,65$ & ${ }^{*}$ & 0,42 \\
\hline Poda a 1 tallo & $-0,62$ & $*$ & 0,38 \\
\hline Poda a 2 tallos & $-0,66$ & ${ }^{*}$ & 0,44 \\
\hline Sin poda & $-0,78$ & $* \star$ & 0,62 \\
\hline
\end{tabular}

Nota: $n s=$ no significativo; ${ }^{*}=$ significativa $(p \leq 0,05) ;{ }^{* \star}=$ altamente significativa $(p \leq 0,01)$.

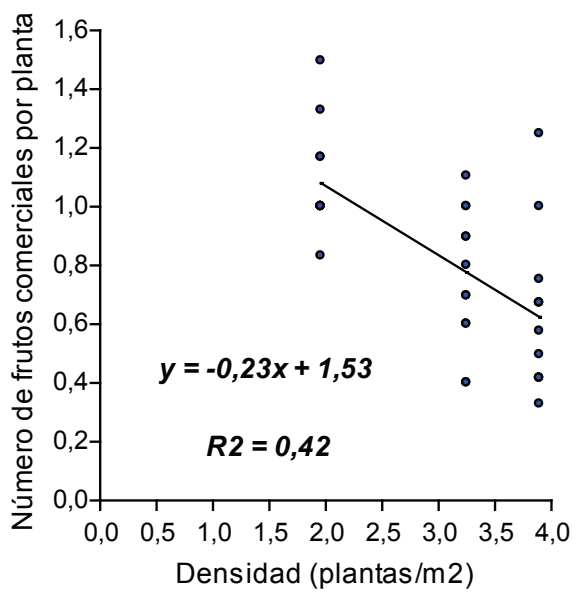

Figura 5. Regresión lineal del número de frutos comerciales por planta versus la densidad de siembra para el total de tratamientos evaluados.

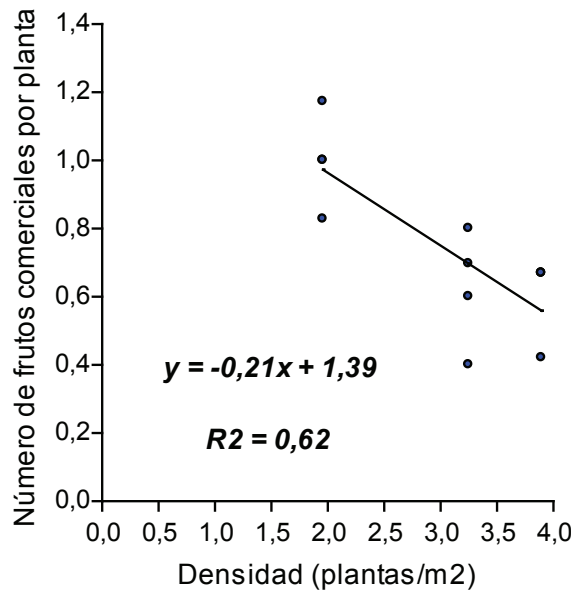

Figura 6. Regresión lineal del número de frutos comerciales por planta versus la densidad de siembra para los tratamientos sin poda. 


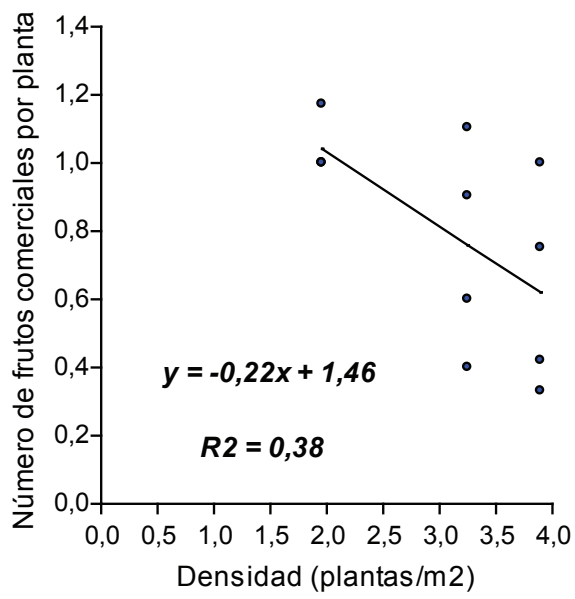

Figura 7. Regresión lineal del número de frutos comerciales por planta versus la densidad de siembra para los tratamientos con poda a 1 tallo.

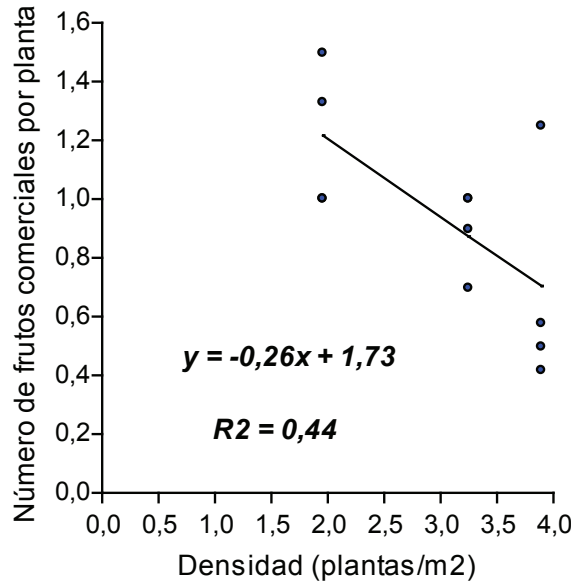

Figura 8. Regresión lineal del número de frutos comerciales por planta versus la densidad de siembra para los tratamientos con poda a 2 tallos.

En cuanto a la correlación entre la densidad de siembra y el rendimiento total por planta, el valor hallado fue negativo y altamente significativo para el total de tratamientos $\left(r=-0,69 ; R^{2}\right.$ $=0,48$ ) (cuadro 4, figura 9), así como para los tratamientos de poda a 2 tallos $\left(r=-0,69 ; R^{2}=\right.$ $0,47)$ (figura 10) y sin poda $\left(r=-0,85 ; R^{2}=0,73\right.$ ) (figura 11); y fue significativo para la poda a 1 tallo $\left(r=-0,62 ; R^{2}=0,38\right)$ (figura 12$)$.

Otros investigadores también hallaron un menor rendimiento total por planta en melón, al aumentar la densidad de siembra [17], al igual que los resultados obtenidos en el presente ensayo.

Cuadro 4. Coeficientes de correlación de Pearson entre densidad de siembra y rendimiento total por planta $(\mathrm{g})$ en melón Honey Dew.

\begin{tabular}{|c|c|c|c|}
\hline Tratamiento & $\begin{array}{c}\text { Coeficiente de } \\
\text { correlación }(r)\end{array}$ & Probabilidad $(\mathrm{p})$ & $\begin{array}{c}\text { Coeficiente de determinación de la } \\
\text { regresión lineal }\left(\mathrm{R}^{2}\right)\end{array}$ \\
\hline Total & $-0,69$ & $* *$ & 0,48 \\
\hline Poda a 1 tallo & $-0,62$ & $*$ & 0,38 \\
\hline Poda a 2 tallos & $-0,69$ & $* *$ & 0,47 \\
\hline Sin poda & $-0,85$ & $* *$ & 0,73 \\
\hline
\end{tabular}

Nota: $n s=$ no significativo; ${ }^{*}=$ significativa $(p \leq 0,05) ;{ }^{* \star}=$ altamente significativa $(p \leq 0,01)$. 


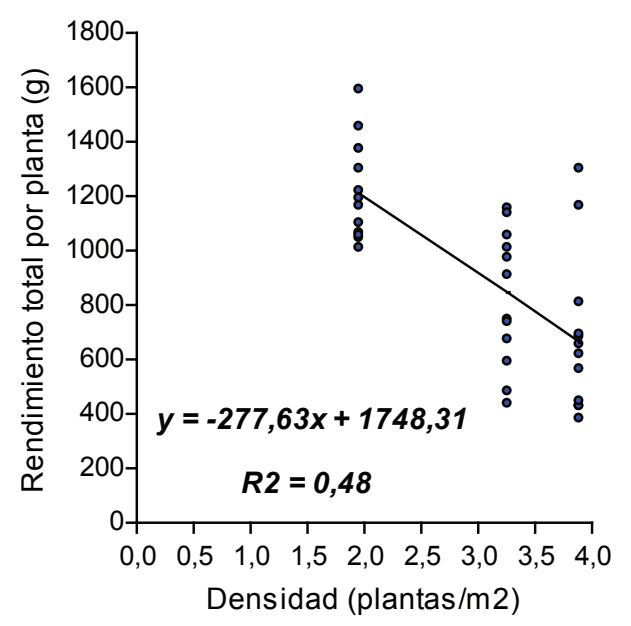

Figura 9. Regresión lineal del rendimiento total por planta versus la densidad de siembra para el total de tratamientos evaluados.

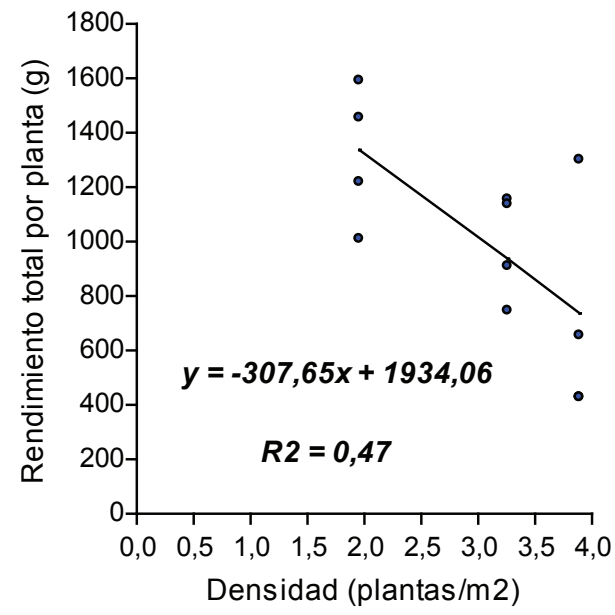

Figura 10. Regresión lineal del rendimiento total por planta versus la densidad de siembra para los tratamientos con poda a 2 tallos.

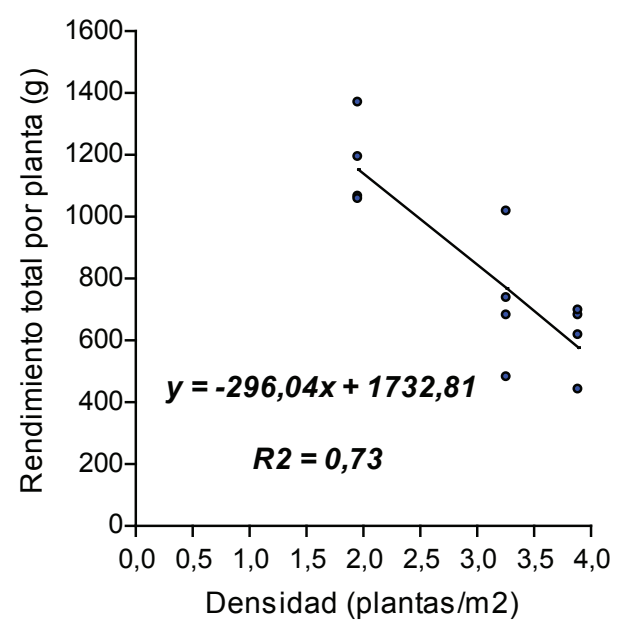

Figura 11. Regresión lineal del rendimiento total por planta versus la densidad de siembra para los tratamientos sin poda. 


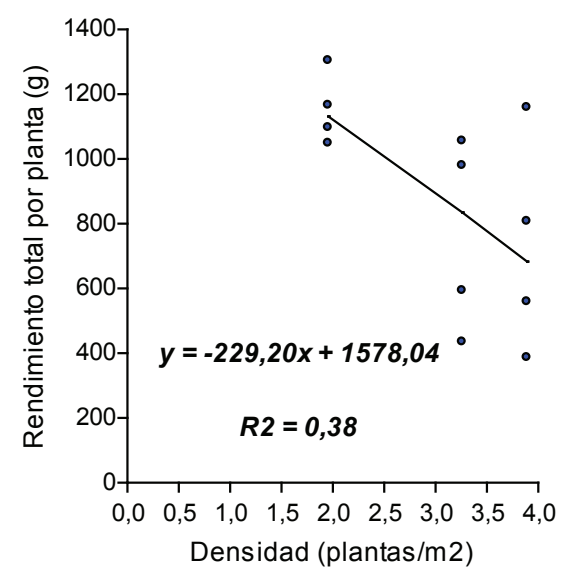

Figura 12. Regresión lineal del rendimiento total por planta versus la densidad de siembra para los tratamientos con poda a 1 tallo.

Con respecto a la correlación entre la densidad de siembra y el rendimiento comercial por planta, el valor obtenido fue negativo y altamente significativo para el total de tratamientos $(r=$ $-0,69 ; R^{2}=0,48$ ) (cuadro 5, figura 13), así como para los tratamientos con poda a 2 tallos $(r=$ $\left.-0,71 ; R^{2}=0,50\right)$ (figura 14) y sin poda $\left(r=-0,87 ; R^{2}=0,76\right)$ (figura 15); y fue significativo para la poda a 1 tallo $\left(r=-0,59 ; R^{2}=0,35\right)$ (figura 16).

Cuadro 5. Coeficientes de correlación de Pearson entre densidad de siembra y rendimiento comercial por planta (g) en melón Honey Dew.

\begin{tabular}{|c|c|c|c|}
\hline Tratamiento & $\begin{array}{c}\text { Coeficiente de } \\
\text { correlación }(r)\end{array}$ & Probabilidad $(\mathrm{p})$ & $\begin{array}{c}\text { Coeficiente de determinación de la } \\
\text { regresión lineal }\left(\mathrm{R}^{2}\right)\end{array}$ \\
\hline Total & $-0,69$ & $* *$ & 0,48 \\
\hline Poda a 1 tallo & $-0,59$ & $*$ & 0,35 \\
\hline Poda a 2 tallos & $-0,71$ & $* \star$ & 0,50 \\
\hline Sin poda & $-0,87$ & $* *$ & 0,76 \\
\hline
\end{tabular}

Nota: $n s=$ no significativo; ${ }^{*}=$ significativa $(p \leq 0,05) ;{ }^{* \star}=$ altamente significativa $(p \leq 0,01)$.

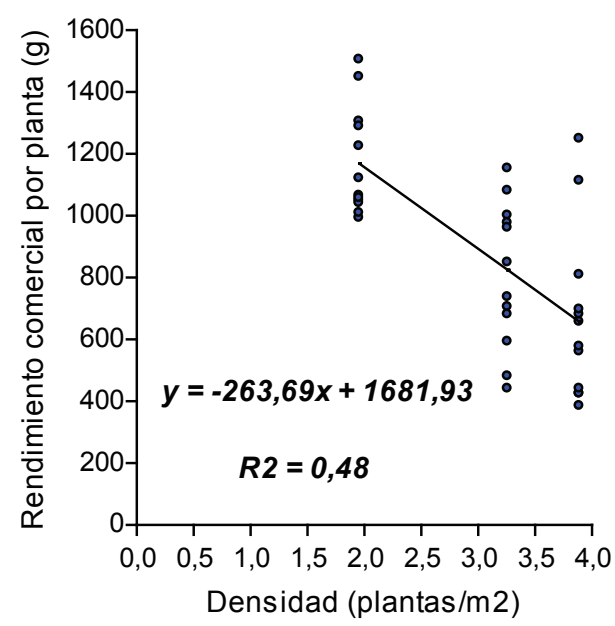

Figura 13. Regresión lineal del rendimiento comercial por planta versus la densidad de siembra para el total de tratamientos evaluados. 


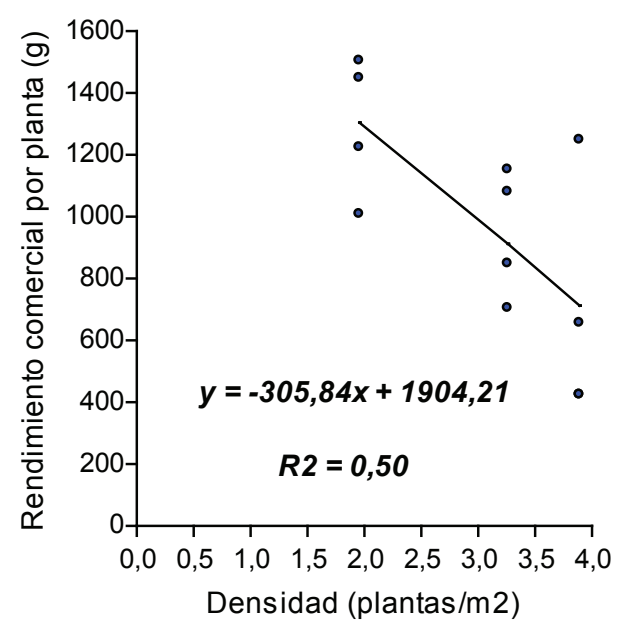

Figura 14. Regresión lineal del rendimiento comercial por planta versus la densidad de siembra para los tratamientos con poda a 2 tallos.

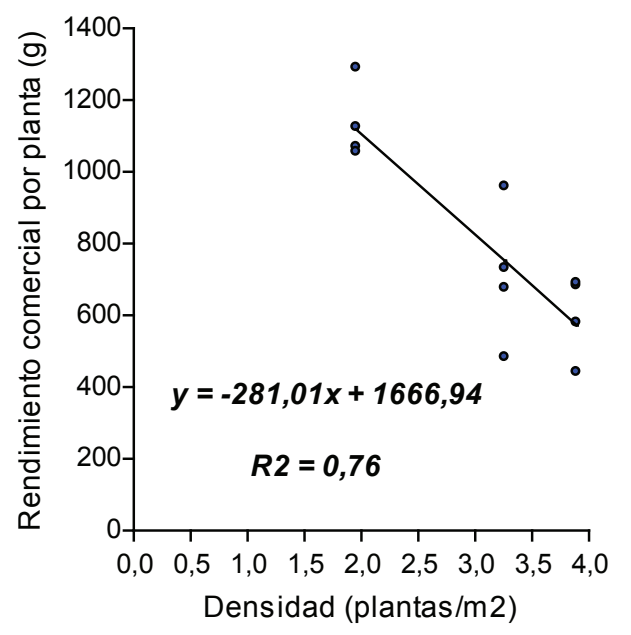

Figura 15. Regresión lineal del rendimiento comercial por planta versus la densidad de siembra para los tratamientos sin poda.

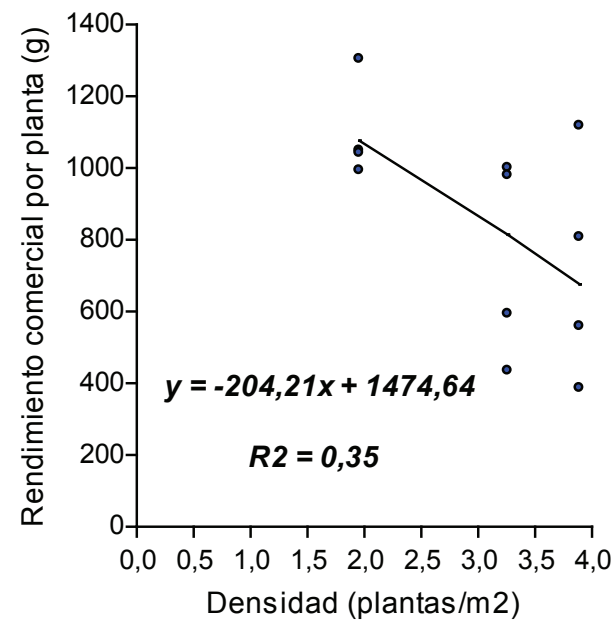

Figura 16. Regresión lineal del rendimiento comercial por planta versus la densidad de siembra para los tratamientos con poda a 1 tallo. 
La correlación entre la densidad de siembra y el porcentaje de sólidos solubles totales fue negativa y significativa para el tratamiento con poda a 2 tallos $\left(r=-0,64 ; R^{2}=0,41\right)$ (cuadro 6 , figura 17); pero fue no significativa para el total de tratamientos, y para los tratamientos con poda a 1 tallo y sin poda.

Cuadro 6. Coeficientes de correlación de Pearson entre densidad de siembra y porcentaje de sólidos solubles totales ( ${ }^{\circ}$ Brix) en melón Honey Dew.

\begin{tabular}{|c|c|c|c|}
\hline Tratamiento & $\begin{array}{c}\text { Coeficiente de } \\
\text { correlación }(r)\end{array}$ & Probabilidad $(p)$ & $\begin{array}{c}\text { Coeficiente de determinación de la } \\
\text { regresión lineal }\left(R^{2}\right)\end{array}$ \\
\hline Total & $-0,25$ & $n s$ & - \\
\hline Poda a 1 tallo & $-0,13$ & $n s$ & - \\
\hline Poda a 2 tallos & $-0,64$ & $\star$ & 0,41 \\
\hline Sin poda & $-0,06$ & $n s$ & - \\
\hline
\end{tabular}

Nota: $n s=$ no significativo; ${ }^{*}=$ significativa $(p \leq 0,05) ;{ }^{\star \star}=$ altamente significativa $(p \leq 0,01)$.

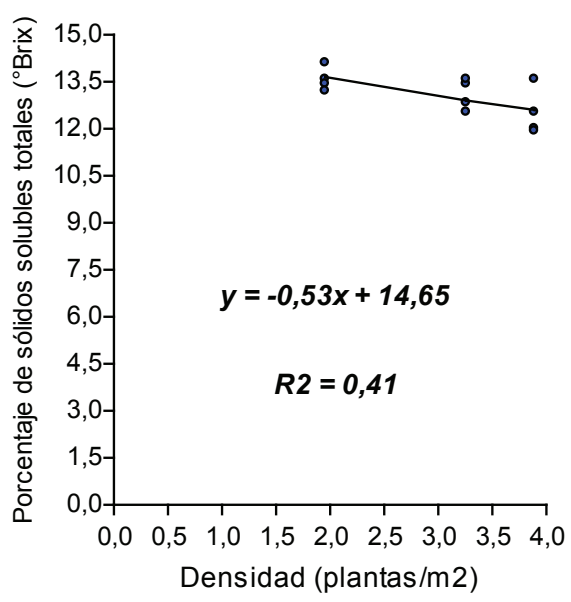

Figura 17. Regresión lineal del porcentaje de sólidos solubles totales versus la densidad de siembra para los tratamientos con poda a 2 tallos.

La disminución en los valores obtenidos en el porcentaje de sólidos solubles totales, conforme aumentó la densidad de siembra en el tratamiento con poda a 2 tallos, es similar a lo encontrado por varios investigadores [19] [20] [21]. El incremento en el contenido de carbohidratos ('Brix) está relacionado con el incremento en el área foliar, como resultado de una mayor separación entre plantas (menor densidad de siembra), es decir, una menor competencia entre ellas [21]. Sin embargo, otros autores no encontraron diferencias significativas para esta variable al evaluar diferentes densidades de siembra [14] [13] [17]; y otros investigadores hallaron que, para un híbrido de melón, el porcentaje de sólidos solubles totales aumentó al incrementarse la densidad de siembra [21].

En cuanto a la correlación entre la densidad de siembra y la edad al inicio de la cosecha, el valor hallado fue positivo y altamente significativo para el total de tratamientos $\left(r=0,55 ; R^{2}=\right.$ 0,30 ) (cuadro 7, figura 18), así como para el tratamiento sin poda $\left(r=0,98 ; R^{2}=0,96\right.$ ) (figura 19); pero fue no significativo para los tratamientos con poda a 1 tallo y a 2 tallos. 
Otros investigadores no encontraron diferencias en la edad al inicio de la cosecha, al evaluar diferentes densidades de siembra en melón tipo Cantaloupe [19] [22].

Cuadro 7. Coeficientes de correlación de Pearson entre densidad de siembra y edad al inicio de la cosecha (ddt) en melón Honey Dew.

\begin{tabular}{|c|c|c|c|}
\hline Tratamiento & $\begin{array}{c}\text { Coeficiente de } \\
\text { correlación }(r)\end{array}$ & Probabilidad $(p)$ & $\begin{array}{c}\text { Coeficiente de determinación de la } \\
\text { regresión lineal }\left(R^{2}\right)\end{array}$ \\
\hline Total & 0,55 & $* \star$ & 0,30 \\
\hline Poda a 1 tallo & 0,55 & $n s$ & - \\
\hline Poda a 2 tallos & 0,55 & $n s$ & 0,96 \\
\hline Sin poda & 0,98 & $* \star$ & - \\
\hline
\end{tabular}

Nota: ns $=$ no significativo $; *=$ significativa $(\mathrm{p} \leq 0,05) ; * *=$ altamente significativa $(\mathrm{p} \leq 0,01)$.

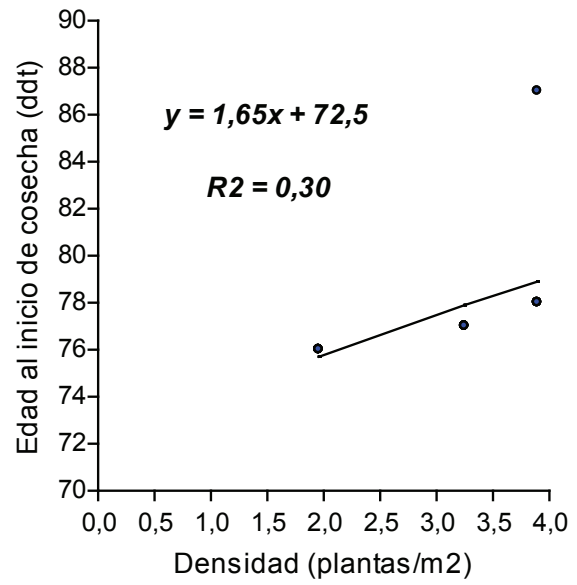

Figura 18. Regresión lineal de la edad al inicio de la cosecha versus la densidad de siembra para el total de tratamientos evaluados.

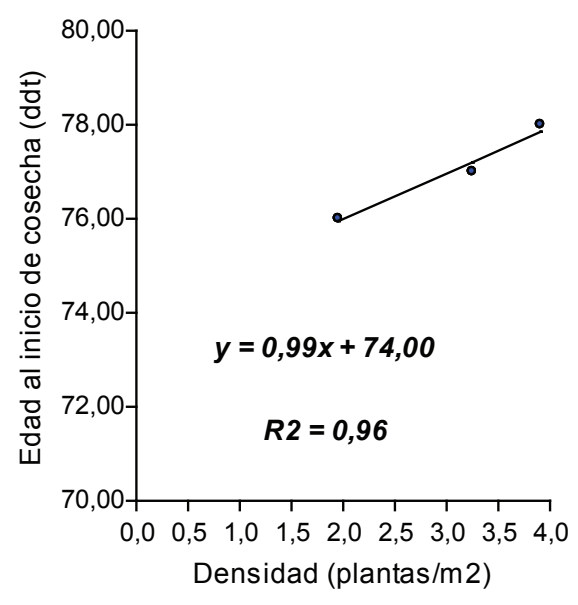

Figura 19. Regresión lineal de la edad al inicio de la cosecha versus la densidad de siembra para los tratamientos sin poda. 
En todos los casos, las correlaciones fueron no significativas, entre la densidad de siembra y las siguientes variables: peso del fruto (cuadro 8); rendimiento por área (cuadro 9); firmeza de la pulpa del fruto (cuadro 10); y número total de frutos por metro cuadrado (cuadro 11).

Cuadro 8. Coeficientes de correlación de Pearson entre densidad de siembra y peso del fruto (g) en melón Honey Dew.

\begin{tabular}{|c|c|c|c|}
\hline Tratamiento & $\begin{array}{c}\text { Coeficiente de } \\
\text { correlación }(r)\end{array}$ & Probabilidad $(p)$ & $\begin{array}{c}\text { Coeficiente de determinación de la } \\
\text { regresión lineal }\left(R^{2}\right)\end{array}$ \\
\hline Total & $-0,03$ & $n s$ & - \\
\hline Poda a 1 tallo & 0,50 & $n s$ & - \\
\hline Poda a 2 tallos & $-0,32$ & $n s$ & - \\
\hline Sin poda & $-0,32$ & $n s$ & ns \\
\hline
\end{tabular}

Nota: $n s=$ no significativo; ${ }^{*}=$ significativa $(p \leq 0,05) ;{ }^{* \star}=$ altamente significativa $(p \leq 0,01)$.

Cuadro 9. Coeficientes de correlación de Pearson entre densidad de siembra y rendimiento por área (ton/ha) en melón Honey Dew.

\begin{tabular}{|c|c|c|c|}
\hline Tratamiento & $\begin{array}{c}\text { Coeficiente de } \\
\text { correlación }(r)\end{array}$ & Probabilidad $(\mathrm{p})$ & $\begin{array}{c}\text { Coeficiente de determinación de la } \\
\text { regresión lineal }\left(\mathrm{R}^{2}\right)\end{array}$ \\
\hline Total & 0,19 & $\mathrm{~ns}$ & - \\
\hline Poda a 1 tallo & 0,30 & $\mathrm{~ns}$ & - \\
\hline Poda a 2 tallos & 0,15 & $\mathrm{~ns}$ & - \\
\hline Sin poda & 0,14 & $\mathrm{~ns}$ & - \\
\hline
\end{tabular}

Nota: $\mathrm{ns}=$ no significativo; ${ }^{*}=$ significativa $(\mathrm{p} \leq 0,05) ;{ }^{* \star}=$ altamente significativa $(\mathrm{p} \leq 0,01)$.

Cuadro 10. Coeficientes de correlación de Pearson entre densidad de siembra y firmeza de la pulpa del fruto $(\mathrm{N})$ en melón Honey Dew.

\begin{tabular}{|c|c|c|c|}
\hline Tratamiento & $\begin{array}{c}\text { Coeficiente de } \\
\text { correlación }(r)\end{array}$ & Probabilidad $(p)$ & $\begin{array}{c}\text { Coeficiente de determinación de la } \\
\text { regresión lineal }\left(R^{2}\right)\end{array}$ \\
\hline Total & 0,09 & ns & - \\
\hline Poda a 1 tallo & 0,05 & $n s$ & - \\
\hline Poda a 2 tallos & 0,17 & ns & - \\
\hline Sin poda & 0,25 & ns & - \\
\hline
\end{tabular}

Nota: $n s=$ no significativo; ${ }^{*}=$ significativa $(p \leq 0,05) ;{ }^{*}=$ altamente significativa $(p \leq 0,01)$.

Cuadro 11. Coeficientes de correlación de Pearson entre densidad de siembra y número total de frutos por $\mathrm{m}^{2}$ en melón Honey Dew.

\begin{tabular}{|c|c|c|c|}
\hline Tratamiento & $\begin{array}{c}\text { Coeficiente de } \\
\text { correlación }(r)\end{array}$ & Probabilidad $(\mathrm{p})$ & $\begin{array}{c}\text { Coeficiente de determinación de la } \\
\text { regresión lineal }\left(\mathrm{R}^{2}\right)\end{array}$ \\
\hline Total & 0,16 & $\mathrm{~ns}$ & - \\
\hline Poda a 1 tallo & 0,12 & $\mathrm{~ns}$ & - \\
\hline Poda a 2 tallos & 0,19 & $\mathrm{~ns}$ & - \\
\hline Sin poda & 0,23 & $\mathrm{~ns}$ & - \\
\hline
\end{tabular}

Nota: $n s=$ no significativo; ${ }^{*}=$ significativa $(p \leq 0,05) ;{ }^{* \star}=$ altamente significativa $(p \leq 0,01)$. 
Por lo tanto, no se presentaron diferencias significativas en el rendimiento total ni comercial por área entre los diferentes tratamientos de densidad de siembra. Estos resultados son contrarios a los obtenidos por otros investigadores, quienes sí hallaron un mayor rendimiento de melón por área al aumentar la densidad de siembra [10] [11] [12] [13] [14].

\section{Conclusiones}

Se concluye que, en melón Honey Dew manejado con cualquiera de los tipos de poda evaluados, el uso de una mayor densidad de siembra provoca una disminución en el número de frutos (totales y comerciales) por planta, y en el rendimiento total y comercial por planta.

\section{Agradecimientos}

Los autores agradecen la colaboración de Cristina Arguedas, Julio Vega, Carlos González y Andrés Oviedo en el trabajo de campo, y de Mario Monge en la revisión de la traducción del resumen al idioma inglés. Asimismo, agradecen el financiamiento recibido por parte de la Vicerrectoría de Investigación de la Universidad de Costa Rica para la realización de este trabajo.

\section{Referencias}

[1] J. M. Torres, «Los tipos de melón comerciales,» de Melones, España, Ediciones de Horticultura, 1997, pp. 13-20.

[2] J. Reche, Cultivo intensivo del melón, Madrid, España: Ministerio de Agricultura, Pesca y Alimentación, 2007.

[3] J. E. Monge-Pérez, «Producción y exportación de melón (Cucumis melo) en Costa Rica,» Tecnología en Marcha, vol. 27, n 1, pp. 93-103, 2014.

[4] P. F. Vargas, R. Castoldi, H. C. O. Charlo y L. T. Braz, «Qualidade de melão rendilhado (Cucumis melo L.) em função do sistema de cultivo,» Ciência e Agrotecnologia, vol. 32, nº 1, pp. 137-142, 2008.

[5] S. R. Martins, R. M. Peil, J. E. Schwengber, F. N. Assis y M. E. G. Mendez, «Produção de melão em função de diferentes sistemas de condução de plantas em ambiente protegido,» Horticultura Brasileira, vol. 16, n 1, pp. 24-30, 1998.

[6] J. E. Monge-Pérez, «Evaluación preliminar de 201 genotipos de ocho diferentes hortalizas (berenjena, chile dulce, zucchini, ayote, sandía, pepino, tomate y melón) cultivados bajo invernadero en Costa Rica,» de La investigación en Guanacaste II, San José, Costa Rica, Editorial Nuevas Perspectivas, 2016, pp. 277-300.

[7] B. Santos, H. Obregón-Olivas y T. Salamé-Donoso, «Producción de hortalizas en ambientes protegidos: estructuras para la agricultura protegida,» University of Florida, IFAS Extension, UF Department of Horticultural Sciences, Florida, Estados Unidos, 2010.

[8] T. Alvarado-Sánchez y J. E. Monge-Pérez, «Efecto de la aplicación de bioactivadores y del raleo manual de frutos sobre el rendimiento y la calidad de melón (Cucumis melo L.) bajo cultivo protegido en Costa Rica,» Tecnología en Marcha, vol. 28, n 4, pp. 15-25, 2015.

[9] M. L. Gómez-Guillamón, R. Camero y J. Gonzáles-Fernández, «El melón en invernadero,» de Melones, Barcelona, España, Ediciones de Horticultura S. L., 1997, pp. 67-77.

[10] H. Nerson, «Effects of population density on fruit and seed production in muskmelons,» Acta Horticulturae, vol. 492, pp. 65-70, 1999.

[11] F. H. Pereira, I. Nogueira, J. Pedrosa, M. Negreiros y F. Bezerra-Neto, «Poda da haste principal e densidade de cultivo na produção e qualidade de frutos em híbridos de melão,» Horticultura Brasileira, vol. 21, n 2, pp. 191-196, 2003.

[12] D. Ban, S. Goreta y J. Borosic, «Plant spacing and cultivar affect melon growth and yield components," Scientia Horticulturae, vol. 109, pp. 238-243, 2006.

[13] J. C. Rodríguez, N. L. Shaw y D. J. Cantliffe, «Influence of plant density on yield and fruit quality of greenhousegrown galia muskmelons,» Hort Technology, vol. 17, n 4, pp. 580-585, 2007. 
[14] J. M. Díaz-Alvarado y J. E. Monge-Pérez, «Producción de melón (Cucumis melo L.) en invernadero: efecto de poda y densidad de siembra,» Revista Posgrado y Sociedad, vol. 15, n 1, pp. 1-12, 2017.

[15] FAO, El cultivo protegido en clima mediterráneo, Vols. \%1 de \%2Estudios FAO Producción y Protección Vegetal, No. 90, Roma, Italia: Organización de las Naciones Unidas para la Agricultura y la Alimentación, 2002.

[16] J. E. Monge-Pérez y M. Loría-Coto, «Melón (Cucumis melo L.) cultivado bajo invernadero: correlaciones entre variables,» Tecnología en Marcha, vol. 32, nº 1, pp. 134-150, 2019.

[17] F. Kultur, H. C. Harrison y J. E. Staub, «Spacing and genotype affect fruit sugar concentration, yield, and fruit size of muskmelon,» HortScience, vol. 36, n² 2, pp. 274-278, 2001.

[18] J. C. García, Z. F. Rodríguez y J. G. Lugo, «Efecto del cultivar y la distancia entre plantas sobre el comportamiento agronómico y rendimiento del melón,» Revista de la Facultad de Agronomía (LUZ), vol. 23, pp. 443452, 2006.

[19] J. M. Díaz-Alvarado y J. E. Monge-Pérez, «Efecto de la poda y la densidad de siembra sobre el rendimiento y calidad de melón Cantaloupe (Cucumis melo L.) cultivado bajo invernadero,» Revista Colombiana de Ciencias Hortícolas, vol. 11, n 1, pp. 21-29, 2017.

[20] H. Nerson, «Relationship between plant density and fruit and seed production in muskmelon,» Journal of the American Society for Horticultural Science, vol. 127, nº 5, pp. 855-859, 2002.

[21] J. C. García, Z. F. Rodríguez, J. G. Lugo y V. Rodríguez, «Efecto del cultivar y distancia entre plantas sobre características físico-químicas del fruto del melón (Cucumis melo L.), Revista de la Facultad de Agronomía (LUZ), vol. 26, pp. 141-158, 2009.

[22] D. Casaroli, E. B. Fagan, O. S. Santos, D. C. Garcia, S. Sinchak y C. Riffel, «Influência do espaçamento e densidade de frutos por planta em meloeiro hidropônico," Horticultura Brasileira, vol. 22, n 2, Suplemento 2, pp. 1-6, 2004. 